\title{
Outcomes of Unstable Subaxial Cervical Spine Fractures Managed by Posteroanterior Stabilization and Fusion
}

\author{
Charanjit Singh Dhillon, Mithun Shriniwas Jakkan, Rishi Dwivedi, \\ Narendra Reddy Medagam, Pankaj Jindal, Shrikant Ega \\ Department of Spine Services, MIOT International, Chennai, India
}

\begin{abstract}
Study Design: Retrospective study.
Purpose: To evaluate clinical and radiological outcomes of unstable subaxial cervical spine injuries managed by both posterior tension band column stabilization and anterior decompression, stabilization, and fusion.

Overview of Literature: Unstable subaxial cervical spine injuries often involve disruption of the anterior column and posterior tension band osteoligamentous complex. Such injuries need immediate surgical intervention. Different methods of reduction and surgical approaches have been published in the literature, with lack of consensus on a uniform or standardized method. Controversy still exists regarding stabilization of unstable cervical fractures by anterior or posterior approach alone or combined approaches.

Methods: We retrospectively evaluated 24 patients with post-traumatic unstable subaxial cervical spine injuries with their preoperative clinical details, X-ray, computed tomography, and magnetic resonance imaging of the cervical spine for fracture classification based on the mechanism of injury with status of disc herniation and posterior tension band disruption. All patients were managed by immediate reduction, posterior and anterior stabilization, and fusion in a single session of anesthesia. Data of all patients were anaIyzed with respect to pre- and postoperative neurological status based on American Spinal Injury Association grading, Visual Analog Scale score, the observation of bony fusion, and implant failure at 1, 3, 6, and 12 months. Data were analyzed using paired $t$-test. Results: All patients had solid fusion at the desired level with considerable neurological improvement at the 1-year follow-up. Conclusions: In unstable cervical injuries, stabilization of disrupted posterior tension band increases the stability of anterior plating and fusion. This method of immediate reduction and circumferential stabilization is rapid, safe, and effective and has a low rate of complications.
\end{abstract}

Keywords: Cervical injuries; Posterior tension band column; Circumferential stabilization

\section{Introduction}

Lower cervical spine injuries account for approximately
$65 \%$ of all cervical spine injuries [1]. Of these, fracture dislocations are the devastating type that most commonly result after motor vehicle accidents (MVAs). These are

Received May 23, 2017; Revised Sep 23, 2017; Accepted Oct 10, 2017

Co-corresponding author: Charanjit Singh Dhillon

Department of Spine Services, MIOT International, 4/112, Mount Ponamallee Road, Manapakkam, Chennai 600089, Tamil Nadu, India Tel: +91-9444518051, Fax: +91-4422491188, E-mail: drdhillonc@hotmail.com

Mithun Shriniwas Jakkan

Department of Spine Services, MIOT International, 4/112, Mount Ponamallee Road, Manapakkam, Chennai 600089, Tamil Nadu, India Tel: +91-8767273585, Fax: +91-4422491313, E-mail: mithunjakkan@gmail.com, drmithunfnb@gmail.com 
highly unstable and often involve disruption of the anterior column and posterior tension band osteoligamentous complex. The incidence of neurological damage is high with these types of fractures. The treatment of choice is often surgical. Such injuries need immediate decompression, reduction, and realignment of cervical spine, with maintenance of reduction through instrumentation by a proper surgical technique and approach. However, management remains controversial despite advances in classification of cervical spine injuries. In this study, we aimed to evaluate the clinical and radiological outcomes of patients with cervical spine injuries managed surgically by both anterior column and posterior osteoligamentous tension band stabilization and fusion.

\section{Materials and Methods}

We retrospectively evaluated all patients with cervical spine injuries who presented to MIOT International, India from January 2014 to June 2015 (IRB approval no., 2016/012/01). The patients included in the study were those with unstable subaxial cervical spine fractures or fracture dislocations with disruptions of posterior tension band column with or without neurological involvement, with a minimum of 1-year follow-up. The patients with intact posterior tension band column, chronic fractures, pathological fractures, and pre-existing diseases, such as ankylosing spondylitis, as well as those who were lost to follow-up and were unwilling to undergo surgery were excluded from the study.

We enrolled 24 consecutive patients, who fulfilled the inclusion criteria, consisting of 23 men and one woman. All patients were evaluated thoroughly, and their detailed demographic and clinicoradiological data were obtained from the day of admission until the last follow-up. The mean age of presentation was 39 years, with the youngest being 17 years and the oldest being 64 years. MVA was the most common mode of injury.

None of our patients presented within the first 48 hours of injury. The mean duration of presentation was 9 days (range, 2-26 days). Once stabilized, X-ray, computed tomography (CT), and magnetic resonance imaging (MRI) of the cervical spine were performed in all patients. Radiological images were also obtained to eliminate injuries to the dorsal and lumbar spine wherever necessary.

These fractures were classified based on the mechanism of injury suggested by Allen et al. [2] The patients includ- ed in this study had disruption in both anterior column and posterior osteoligamentous (tension band) column. The C6-7 level was the most commonly injured level $(\mathrm{n}=10,41.6 \%)$, followed by the C5-6 $(\mathrm{n}=8,33.3 \%), \mathrm{C} 4-5$ $(\mathrm{n}=3,12.5 \%)$, and C7-D1 $(\mathrm{n}=1,4.1 \%)$ levels. Two patients had involvement at two levels: C5-7 and C6-D1 vertebral levels.

The preoperative neurological status was noted and graded based on the American Spinal Injury Association (ASIA: ASIA-A, 11 cases; ASIA-C, one case; ASIA-E, nine cases) grading. Two patients presented with single-root injuries, and one patient presented with central cord syndrome. Associated injuries were also documented in three patients: one with head injury (cerebral concussion), one with open-knee injury, and one with minimally displaced clavicle fracture.

All patients were evaluated and operated at a tertiary care center by a single experienced spine surgeon. The surgery aimed to decompress the cord, reduce fracture dislocation, maintain spinal alignment, and attain rigid and circumferential stabilization of the anterior and posterior column with solid fusion in the same session of anesthesia. Standard Smith-Robinson and vertical midline approaches were used for all anterior and posterior procedures, respectively.

All patients with facet subluxation or dislocation were managed with posterior reduction and stabilization followed by anterior column reconstruction and fusion using graft obtained from autologous iliac crest bone graft. Eight patients either had an extruded disc fragment $(\mathrm{n}=2)$ or a tear drop fracture $(n=6)$ with larger retropulsion fragment compressing the cord. In these patients, anterior decompression and fusion by bone graft obtained on corpectomy bone and cage was initially performed, followed by posterior tension band stabilization.

All patients were monitored in the intensive care unit (ICU) postoperatively. The patients were later mobilized with soft cervical collar for the next 3 months. Wheel chair mobilization was performed for patients with power $<3 / 5$ in the lower limbs, and parallel bar mobilization with knee splints to prevent knee buckling was performed for those with power $>3 / 5$. All patients underwent aggressive physiotherapy up to 1 year.

Patients were followed up at 1, 3, and 6 months and 1 year postoperatively. They were evaluated clinically for neck pain (Visual Analogue Scale score) and discomfort and neurological recovery. Cervical spine X-ray was ob- 


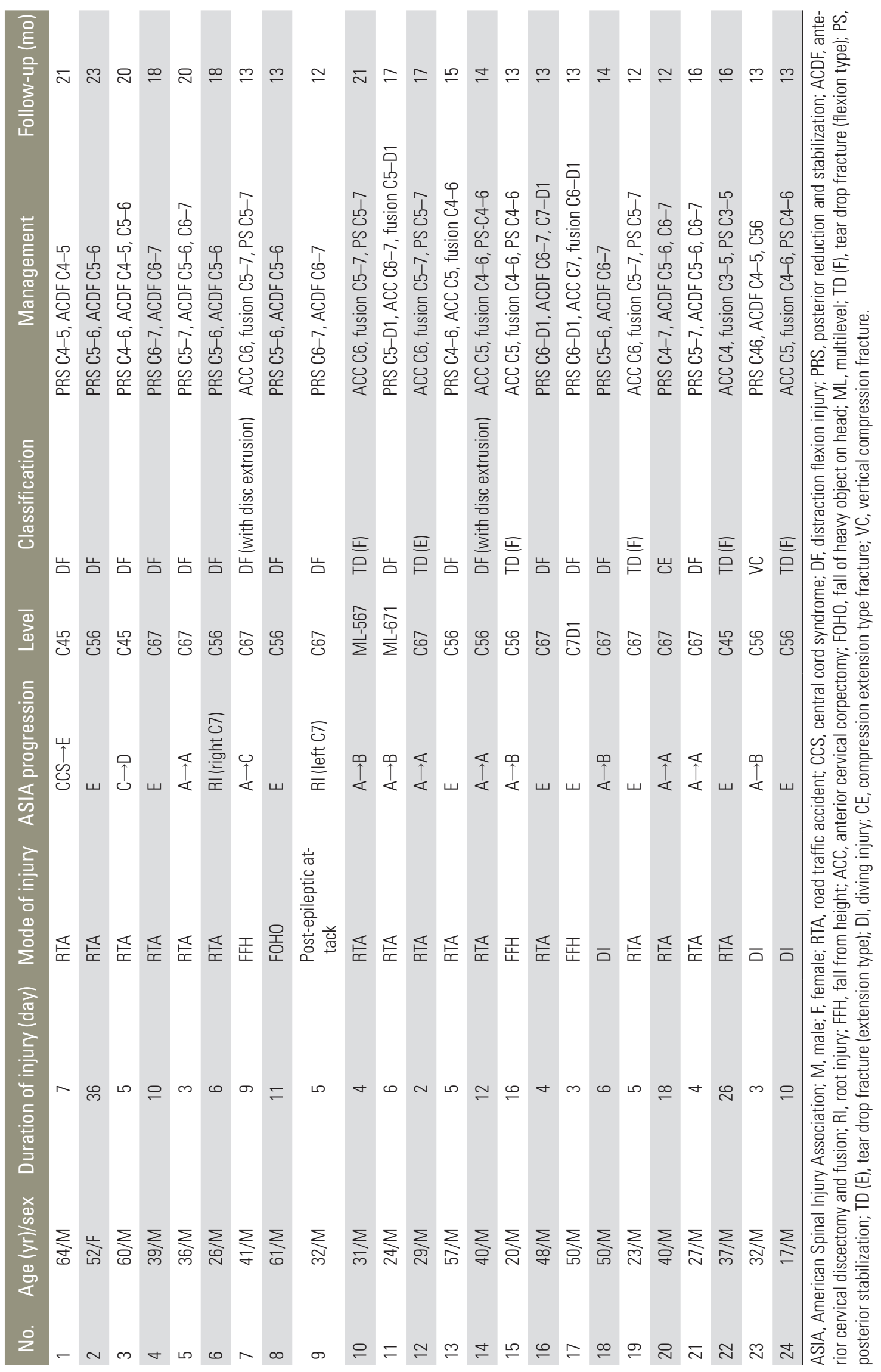


tained in each follow-up and evaluated for spine alignment and any implant-related complications. At the end of 1 year, fusion at desired site was evaluated by $\mathrm{X}$-ray alone in patients showing obvious solid fusion with trabecular continuity and by CT scan in patients where confirmation of fusion was needed, particularly in cases where a cage was used. The duration of the follow-up of the patients ranged from 13 months to 23 months, with a mean of 15.2 months.

\section{Results}

All patients underwent reduction and stabilization by anterior and posterior approach in a single session of anesthesia (Table 1). No patient deteriorated after completing the surgical procedure. Most of the patients had uneventful postoperative stay, except for six patients. Of six patients, three had respiratory tract infection, which was treated by appropriate intravenous antibiotics, based on their sensitivity report and were eventually cured. One patient had posterior wound dehiscence, which was managed by secondary suturing, and the wound healed by secondary intention. Two patients developed grade 2 sacral sores, and both were cured after conservative management.

There was considerable neurological recovery with respect to the ASIA grade in patients. Two patients, one with right $\mathrm{C} 7$ root and the other with left $\mathrm{C} 7$ root injury, showed improvement of grade 4/5 power, but some amount of paresthesia was still present.

All patients had significant improvement with respect to neck pain $(p<0.05)$. Few patients had neck discomfort and stiffness in the postoperative period. Serial radiographs showed maintained realignment of the cervical spine in the follow-up, with solid fusion at the involved site that was assessed by obtaining X-ray and CT scan of the cervical spine at the end of 1 year. The scans showed complete fusion with continuity of trabecular bone at the operated level in all patients. No hardware failure was noted in any of the patient. Only one patient developed subtle anterior subluxation of the vertebral body above the adjacent level, but he was asymptomatic and was satisfied with the result and hence was managed conservatively (Figs. 1, 2).

\section{Discussion}

Unstable subaxial cervical spine injuries usually occur

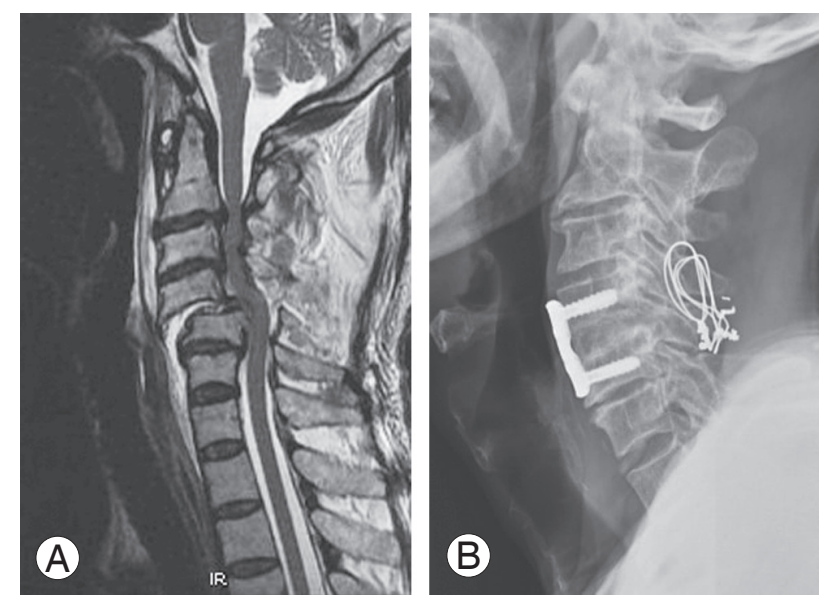

Fig. 1. (A) Magnetic resonance imaging-sagittal view of a 64 -yearold male with flexion distraction injury at the C4-5 level. (B) Postoperative $\mathrm{X}$-ray at 1 year showing reduction and fusion with trabecular continuity at the $\mathrm{C} 4-5$ level.
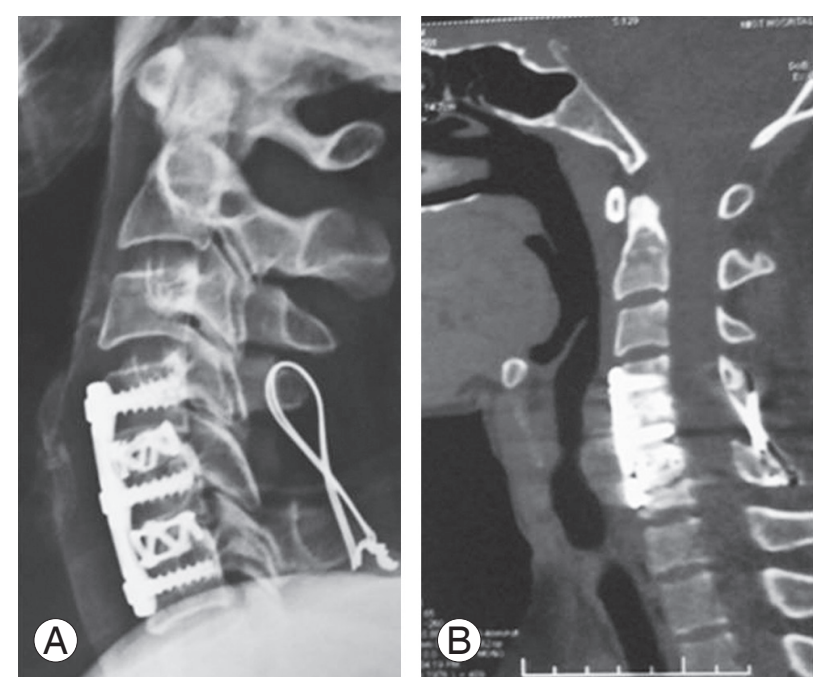

Fig. 2. (A, B) X-ray and computed tomography scan showing good alignment and fusion 13 months postoperatively in a patient with two-level cervical injury.

because of MVA and falling from a height [3-5]. Of $24 \mathrm{pa}-$ tients, 16 of our patients experienced MVAs (66.6\%). Such injuries are often associated with neurological deficit from complete cord injury to radiculopathy [6].

We classified such injuries using the classification system developed by Allen et al. [2] to understand the mechanism of injury and plan the treatment. This classification considers the severity and mechanism of injury, which helps determine an appropriate plan of management $[2,7,8]$, although the system was based (in 1982) on X-ray alone, wherein posterior arch fractures and disco-ligamentous injuries can be missed $[9,10]$. Thus, 
we performed CT and MRI of all patients because CT scan detects $97 \%-100 \%$ of fractures, and MRI is useful in assessing the intervertebral disc status and ligamentous structures and plan the management particularly for extruded disc, initially requiring anterior decompression [8,11-16].

Several other classification systems have been proposed, such as the subaxial cervical spine injury classification system and AO spine classification group $[6,10,17]$. Despite technological advances, classification and treatment of subaxial cervical spine injuries remain controversial $[6,18]$. No injury classification system has currently achieved universal use [9].

Considering the injury level, $75 \%$ of injuries were around the $\mathrm{C} 5$ and $\mathrm{C} 7$ level. Also, in many studies, $>50 \%$ of injuries are located between C5 and C7 [8,12,19]. Of 24 patients, 11 sustained complete cord injury, whereas three had incomplete cord injuries. For all spinal cord injuries (SCIs), the cervical spine remains the most commonly injured level (55\%) [8]. Such injuries are more common in unstable subaxial cervical spine fracture and fracture dislocations [19].

These injuries need to be addressed surgically with the aim of reversing and preventing deterioration of neurological damage, achieving spinal reduction, stabilization, and early mobilization [11]. Different methods of reduction and surgical approaches have been published in the literature that lack consensus on uniform or standardized method $[6,20]$. Controversy still exists regarding the stabilization of unstable cervical fractures by anterior or posterior approach alone or combined approaches [21].

Many studies in the literature are injury morphology specific but lacking validity. Lifeso et al. [22] and Laus et al. [23] treated unstable cervical spine injuries with anterior approach alone with fusion in all their cases. According to Reindl et al. [24], additional posterior surgery should be reserved for irreducible fractures, fracture of posterior elements, and irreducible dislocations or associated with disc extrusion. However, awake closed reduction is possible only if the patient presents within the first 48 hours of injury. This will cause increase in length of immobilization and pain and ventral cord compression in cases owing to herniated disc post-reduction $[25,26]$. Closed reduction usually requires intensive monitoring of the patient in ICU with repeated X-rays to monitor reduction. Because none of our patients presented to us within the first 48 hours of injury, closed reduction was deferred in our patients.

In our study, the most common type was flexion distraction injury, wherein the injury initially originates in the posterior elements and travels to the anterior elements of the cervical spine, making it unstable anteroposteriorly. Hence, a posterior tension band was necessary for stabilization to prevent distraction of posterior elements and to increase stability of anterior implant and cage during flexion motion of the cervical spine. This allows early mobilization of the patient.

We initially used the posterior approach in many cases to achieve direct and complete reduction of facet dislocation, to prevent overdistraction of the anterior column during anterior plating, to excise any loose posterior fractured fragments, such as the lamina, which can be used as bone graft with cage for anterior fusion, thereby preventing the harvesting of autologous graft, and to achieve stability of the anterior plate and prevent the posterior subluxation of the graft during flexion movement of the cervical spine.

We initially used the anterior approach in cases with extruded disc behind the vertebral body and tear drop fractures, and cord decompression was achieved by corpectomy and discectomy with plating and fusion with bone graft obtained from corpectomy (autologous graft) bone and cage. This was followed by posterior stabilization. We achieved complete fusion in all our patients.

Some studies in the literature present with similar principles. Shen et al. [14] reported 19 cases of successful closed reduction under general anesthesia followed by anteroposterior spinal reconstruction. Ngo et al. [18] suggested gentle reduction and posterior spinal fusion alone or combined anterior and posterior spinal fusion for patients with fracture and contralateral dislocation of twin facet joints. In distraction flexion injury stage 3, 4 with loss of posterior tension band complex were instant axis of rotation is located more posterior to anterior construct hence, needs supplementation by posterior fixation [15]. Intact or stable posterior tension band stabilizes flexion motion of the spine and augments anterior cervical instrumentation [27]. However, no significant difference was found in fusion rates, alignment, neurological recovery, or long-term complications of pain in patients treated with either anterior or posterior fusion with instrumentation for unstable cervical injuries [27].

We used different types of implants for posterior stabilization as per the surgeon's choice, indications, and cost 
constraints. None of the cases showed any implant-related complications. In cadaveric studies performed by Ghori et al. [28], dorsal stabilization techniques (i.e., Rogers wiring, sublaminar wiring, Bohlman wiring, Roy Camille dorsal plate fixation, and dorsal hook plate fixation) were compared without any significant biomechanical differences noted. However, wiring techniques are not useful in laminar and spinous process fractures; thus, lateral mass fixation is gaining popularity [28].

The timing of surgery has an important role in spinal cord recovery because emergency surgery within 24 hours for SCI significantly improved the neurologic outcomes compared with late surgery [12,29]. None of our patients presented to us within 48 hours of injury. Despite this, considerable neurological recovery was postoperatively observed in many of our patients.

The advantages of combined approach is to achieve superior biomechanical stability because the injured posterior tension band is stabilized, providing more stability to anterior implants and allowing sound fusion. It gives an opportunity for the complete posterior and anterior decompression of the cervical cord in a single session of anesthesia. It also allows early mobilization of patients with soft collar. The disadvantages of such combined procedure in a single session of anesthesia are the duration of anesthesia, another incision, increased duration of surgery, and more blood loss. However, comparative studies with only anterior/posterior approach, a larger sample size, and a longer follow-up are needed for further validation.

\section{Conclusions}

Highly unstable subaxial cervical spine fractures with anterior column and posterior tension band complex disruption should be managed by immediate reduction, realignment, and circumferential stabilization and fusion in a single session of anesthesia, providing good results in alignment, fusion, and neurological recovery.

\section{Conflict of Interest}

No potential conflict of interest relevant to this article was reported.

\section{References}

1. Song KJ, Lee SK, Ham DH, Kim YJ, Choi BW. Limi- tation of previous Allen classification and subaxial cervical spine injury classification (SLIC) system in distractive-extension injury of cervical spine: proposal of modified classification system. Eur Spine J 2016;25:74-9.

2. Allen BL Jr, Ferguson RL, Lehmann TR, O’Brien RP. A mechanistic classification of closed, indirect fractures and dislocations of the lower cervical spine. Spine (Phila Pa 1976) 1982;7:1-27.

3. Blizzard DJ, Miller CP, Blizzard ST, Grauer JN. Incidence and demographics of cervical spine fractures over a 10 year period at level I trauma center. Duke Orthop J 2016;6: 21-5.

4. Lalwani S, Singh V, Trikha V, et al. Mortality profile of patients with traumatic spinal injuries at a level I trauma care centre in India. Indian J Med Res 2014;140:40-5.

5. Thompson C, Gonsalves JF, Welsh D. Hyperextension injury of the cervical spine with central cord syndrome. Eur Spine J 2015;24:195-202.

6. Shousha M. ABCD classification system: a novel classification for subaxial cervical spine injuries. Spine (Phila Pa 1976) 2014;39:707-14.

7. Liu P, Zhao J, Liu F, Liu M, Fan W. A novel operative approach for the treatment of old distractive flexion injuries of subaxial cervical spine. Spine (Phila $\mathrm{Pa}$ 1976) 2008;33:1459-64.

8. Torretti JA, Sengupta DK. Cervical spine trauma. Indian J Orthop 2007;41:255-67.

9. Urrutia J, Zamora T, Campos M, et al. A comparative agreement evaluation of two subaxial cervical spine injury classification systems: the AOSpine and the Allen and Ferguson schemes. Eur Spine J 2016;25:218592.

10. Vaccaro AR, Hulbert RJ, Patel AA, et al. The subaxial cervical spine injury classification system: a novel approach to recognize the importance of morphology, neurology, and integrity of the disco-ligamentous complex. Spine (Phila Pa 1976) 2007;32:2365-74.

11. O’Dowd JK. Basic principles of management for cervical spine trauma. Eur Spine J 2010;19 Suppl 1:S1822.

12. Joaquim AF, Patel AA. Subaxial cervical spine trauma: evaluation and surgical decision-making. Global Spine J 2014;4:63-70.

13. Crim JR, Moore K, Brodke D. Clearance of the cervical spine in multitrauma patients: the role 
of advanced imaging. Semin Ultrasound CT MR 2001;22:283-305.

14. Shen Y, Shen HL, Feng ML, Zhang WB. Immediate reduction under general anesthesia and single-staged anteroposterior spinal reconstruction for fracturedislocation of lower cervical spine. J Spinal Disord Tech 2015;28:E1-8.

15. Henriques T, Olerud C, Bergman A, Jonsson H Jr. Distractive flexion injuries of the subaxial cervical spine treated with anterior plate alone. J Spinal Disord Tech 2004;17:1-7.

16. Rizzolo SJ, Vaccaro AR, Cotler JM. Cervical spine trauma. Spine (Phila Pa 1976) 1994;19:2288-98.

17. Vaccaro AR, Koerner JD, Radcliff KE, et al. AOSpine subaxial cervical spine injury classification system. Eur Spine J 2016;25:2173-84.

18. Ngo LM, Aizawa T, Hoshikawa T, et al. Fracture and contralateral dislocation of the twin facet joints of the lower cervical spine. Eur Spine J 2012;21:282-8.

19. Koivikko MP, Myllynen P, Santavirta S. Fracture dislocations of the cervical spine: a review of 106 conservatively and operatively treated patients. Eur Spine J 2004;13:610-6.

20. Patel AA, Hurlbert RJ, Bono CM, Bessey JT, Yang $\mathrm{N}$, Vaccaro AR. Classification and surgical decision making in acute subaxial cervical spine trauma. Spine (Phila Pa 1976) 2010;35(21 Suppl):S228-34.

21. Suk KS, Kim KT, Lee JH, Lee SH, Kim JS, Eoh JH. Stage 4 compressive-extension injury of the cervical spine: a theoretical stage? Spine (Phila Pa 1976)
2010;35:E733-8.

22. Lifeso RM, Colucci MA. Anterior fusion for rotationally unstable cervical spine fractures. Spine (Phila $\mathrm{Pa}$ 1976) 2000;25:2028-34.

23. Laus M, Pignatti G, Tigani D, Alfonso C, Giunti A. Anterior decompression and plate fixation in fracture dislocations of the lower cervical spine. Eur Spine J 1993;2:82-8.

24. Reindl R, Ouellet J, Harvey EJ, Berry G, Arlet V. Anterior reduction for cervical spine dislocation. Spine (Phila Pa 1976) 2006;31:648-52.

25. Payer M. Immediate open anterior reduction and antero-posterior fixation/fusion for bilateral cervical locked facets. Acta Neurochir (Wien) 2005;147:50913.

26. Signoret F, Jacquot FP, Feron JM. Reducing the cervical flexion tear-drop fracture with a posterior approach and plating technique: an original method. Eur Spine J 1999;8:110-6.

27. Brodke DS, Anderson PA, Newell DW, Grady MS, Chapman JR. Comparison of anterior and posterior approaches in cervical spinal cord injuries. J Spinal Disord Tech 2003;16:229-35.

28. Ghori A, Le HV, Makanji H, Cha T. Posterior fixation techniques in the subaxial cervical spine. Cureus 2015;7:e338.

29. Liu JM, Long XH, Zhou Y, Peng HW, Liu ZL, Huang $\mathrm{SH}$. Is urgent decompression superior to delayed surgery for traumatic spinal cord injury?: a metaanalysis. World Neurosurg 2016;87:124-31. 